\title{
Exact oscillation regions for a partial difference equation
}

\author{
Chunhua Yuan ${ }^{1,2}$, Shutang Liu ${ }^{1 *}$ and Jian Liu'1,2
}

"Correspondence: stliu@sdu.edu.cn ${ }^{1}$ School of Control Science and Engineering, Shandong University, Jinan, Shandong 250061, P.R. China Full list of author information is available at the end of the article

\begin{abstract}
This paper is concerned with a partial difference equation with constant coefficients. By means of the theory of envelopes, we consider the regions of non-positive roots of its characteristic equation and obtain a necessary and sufficient condition for the oscillation of all solutions of the partial difference equation.
\end{abstract}

MSC: 39A21;39A14

Keywords: partial difference equation; oscillation; envelope; characteristic equation

\section{Introduction}

Partial difference equations have numerous applications in image processing, material mechanics, random walk problems, molecular orbits, finite difference schemes, as well as population dynamics [1-7]. In recent years, the oscillatory behavior of partial difference equations has been discussed in many papers (see [7-14] and the references therein).

In [15-18], Lin and Cheng gave explicit necessary and sufficient conditions for all solutions of some ordinary difference equations with constant coefficients to be oscillatory by means of the envelope theory of a family of straight lines. However, to the best of our knowledge, very little attention has been paid to the analysis of the oscillatory behavior of solutions of partial difference equations from the perspective of the envelope theory.

In this paper, we consider the partial difference equation of the form

$$
u_{n+2, m}+u_{n, m+2}+a u_{n+1, m}+b u_{n, m+1}+c u_{n, m}=0
$$

where $a, b, c$ are real numbers and $m, n$ are nonnegative integers. The purpose of this paper is to develop a new method for the analysis of the oscillation of (1) based on the theory of envelopes.

By a solution of (1), we mean a nontrivial double sequence $\left\{A_{m, n}\right\}$ of real numbers which is defined for $m \geq 0$ and $n \geq 0$ and satisfies (1) for $m \geq 0$ and $n \geq 0$.

A solution $\left\{A_{m, n}\right\}$ of (1) is said to be eventually positive (or negative) if $A_{m, n} \geq 0$ (or $A_{m, n} \leq 0$ ) for large $m$ and $n$. It is said to be oscillatory if it is neither eventually positive nor eventually negative.

The paper is organized as follows. Section 2 presents several useful lemmas. In Section 3, we derive the oscillation criterion for partial difference equation (1). Two examples are given to illustrate our oscillation criterion in Section 4. Finally Section 5 is devoted to concluding remarks. 


\section{Some lemmas}

In this section, we give some preliminaries that will be needed in the next section.

From Corollary 2.9 in [14], we can easily obtain the following lemma.

Lemma 1 The following statements are equivalent:

(a) Every solution of (1) is oscillatory.

(b) The characteristic equation of (1)

$$
\lambda^{2}+\mu^{2}+a \lambda+b \mu+c=0
$$

has no positive roots.

Lemma 2 ([8]) Suppose that $f(x, y), g(x, y), h(x, y)$ and $v(x, y)$ are differentiable on $(-\infty$, $+\infty) \times(-\infty,+\infty)$. Let $\Gamma$ be a two-parameter family of planes defined by the equation

$$
f(\lambda, \mu) x+g(\lambda, \mu) y+h(\lambda, \mu) z=v(\lambda, \mu)
$$

where $\lambda$ and $\mu$ are parameters. Let $\Sigma$ be the envelope of the family $\Gamma$. Then the equation

$$
f(\lambda, \mu) a+g(\lambda, \mu) b+h(\lambda, \mu) c=v(\lambda, \mu)
$$

has no real roots if and only if there is no tangent plane of $\Sigma$ passing through the point $(a, b, c)$ in the Euclidean space $R^{3}$.

Lemma 3 ([19]) For the linear homogeneous difference equation

$$
u_{n+k}+a_{1} u_{n+k-1}+\cdots+a_{k} u_{n}=0
$$

where $n$ is a nonnegative integer, $k$ is a positive integer and $a_{1}, a_{2}, \ldots, a_{k}$ are real numbers, the following statements are equivalent:

(a) Every solution of (2) is oscillatory.

(b) The characteristic equation of (2)

$$
\lambda^{k}+a_{1} \lambda^{k-1}+\cdots+a_{k}=0
$$

has no positive roots.

\section{Main results}

In this section, we establish the necessary and sufficient condition for oscillations of all solutions of (1) by the envelope theory.

Theorem 1 Every solution of (1) oscillates if and only if $a \geq 0, b \geq 0$ and $c \geq 0$ or $a<0$, $b<0$ and

$$
c>\frac{a^{2}+b^{2}}{4} \text {. }
$$


Proof The characteristic equation of (1) is

$$
\lambda^{2}+\mu^{2}+a \lambda+b \mu+c=0
$$

Let

$$
f(a, b, c, \lambda, \mu)=\lambda^{2}+\mu^{2}+a \lambda+b \mu+c=0 .
$$

Since we mainly discuss the oscillations of (1), by Lemma 1, attention will be restricted to the case where $\lambda>0$ and $\mu>0$. To this end, we will think of $(a, b, c)$ as a point in the Euclidean space $R^{3}$ and try to find the exact regions containing points $(a, b, c)$ in the Euclidean space $R^{3}$ such that (3) has no positive roots. In fact, $f(x, y, z, \lambda, \mu)=0$ can be regarded as an equation describing a two-parameter family of planes in the Euclidean space $R^{3}$, where $x, y$ and $z$ are the coordinates of points of the planes in the Euclidean space $R^{3}$ and $\lambda, \mu$ are two parameters.

By the envelope theory, the points of the envelope of the two-parameter family of planes described by (3) satisfy the system of equations

$$
\left\{\begin{array}{l}
f(x, y, z, \lambda, \mu)=\lambda x+\mu y+z+\lambda^{2}+\mu^{2}=0 \\
f_{\lambda}(x, y, z, \lambda, \mu)=x+2 \lambda=0 \\
f_{\mu}(x, y, z, \lambda, \mu)=y+2 \mu=0
\end{array}\right.
$$

where $\lambda>0$ and $\mu>0$. Eliminating the two parameters $\lambda$ and $\mu$ from (4) leads to the equation of the envelope

$$
z(x, y)=\frac{x^{2}+y^{2}}{4}
$$

where $x<0$ and $y<0$. From (5), we have $z(x, y)>0$ for $x<0$ and $y<0, \partial^{2} z / \partial x^{2}=1 / 2>0$, $\partial^{2} z / \partial y^{2}=1 / 2>0$ and $\partial^{2} z / \partial x^{2} \cdot \partial^{2} z / \partial y^{2}-\left(\partial^{2} z / \partial x \partial y\right)^{2}=1 / 4>0$. Hence, $z(x, y)$ is a positive and strictly convex function on $(-\infty, 0) \times(-\infty, 0)$. Furthermore, the envelope defined by $(5)$ is a convex surface $S$ over $(-\infty, 0) \times(-\infty, 0)$ as depicted in Figure 1 . In view of Figure 1 and the obtained information of $z(x, y)$, it is easily seen that when the point $(a, b, c)$ is in the first closed octant, that is, $a \geq 0, b \geq 0$ and $c \geq 0$, there cannot be any tangent plane of the envelope $S$ which also passes through this point, and when the point $(a, b, c)$ is vertically above the envelope $S$, that is, $a<0, b<0$ and $c>\left(a^{2}+b^{2}\right) / 4$, there cannot exist any tangent plane of the envelope $S$ which also passes through this point, while the point $(a, b, c)$ is situated elsewhere, there always exists a tangent plane of the envelope $S$ which also passes through this point. By Lemma 2, the characteristic equation (3) does not have any positive roots if and only if $a \geq 0, b \geq 0$ and $c \geq 0$ or $a<0, b<0$ and

$$
c>\frac{a^{2}+b^{2}}{4} \text {. }
$$

Lemma 1 implies that the statement of this theorem is true. The proof is thus completed. 
Figure 1 Envelope surface for $z(x, y)=\left(x^{2}+y^{2}\right) / 4$ $(x<0, y<0)$.

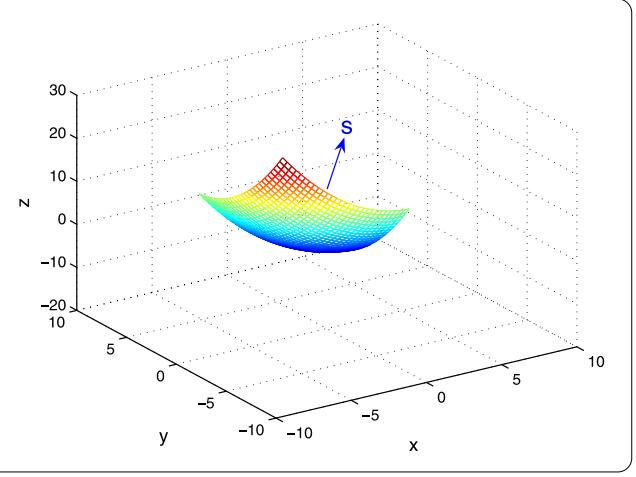

If we let $\mu=0$ in (3), then (3) reduces to the form

$$
\lambda^{2}+a \lambda+c=0
$$

The corresponding ordinary difference equation of (6) takes the form

$$
u_{n+2}+a u_{n+1}+c u_{n}=0 \text {. }
$$

On the basis of Theorem 1, we can obtain the following results.

Corollary 1 Equation (6) has no positive roots if and only if $c \geq 0$ and $a \geq 0$ or $a<0$ and $c>a^{2} / 4$.

Remark 1 In fact, Corollary 1 is just Theorem 5.1 of [20].

Corollary 2 Every solution of (7) oscillates if and only if $c \geq 0$ and $a \geq 0$ or $a<0$ and $c>a^{2} / 4$.

Proof Combining the results of Corollary 1 and Lemma 3 yields the proof.

Remark 2 Corollary 2 shows that Theorem 1 is a natural generalization for the corresponding result of ordinary difference equation (7), and ordinary difference equation (7) is a special case of our study.

\section{Illustrative examples}

In this section, two examples are given to illustrate the applications of Theorem 1.

Example 1 Consider the partial difference equation

$$
u_{n+2, m}+u_{n, m+2}-0.3 u_{n+1, m}-0.1 u_{n, m+1}+0.28 u_{n, m}=0
$$

where $m, n$ are nonnegative integers.

From (8), we have $a=-0.3<0, b=-0.1<0$ and $\left(a^{2}+b^{2}\right) / 4=\left((-0.3)^{2}+(-0.1)^{2}\right) / 4=$ $0.025<0.28=c$. By Theorem 1 , every solution of $(8)$ is oscillatory. The oscillatory behavior of (8) is demonstrated by Figure 2. 


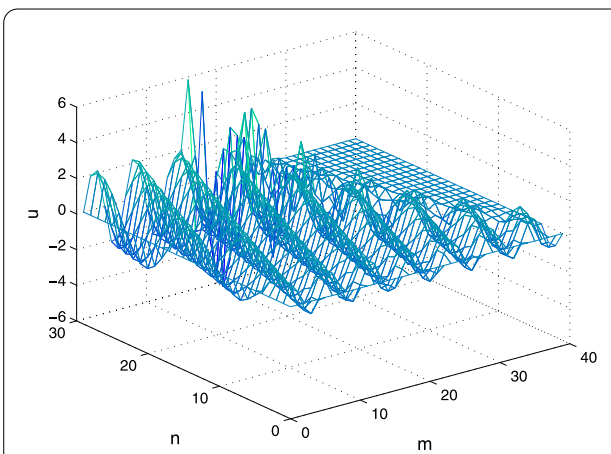

(a) Oscillatory behavior of (8)

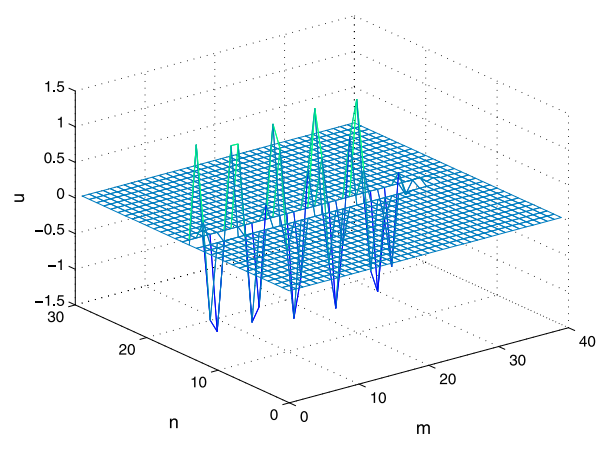

(b) Oscillatory behavior of (8) with $n=15$

Figure 2 Oscillatory behavior of (8).

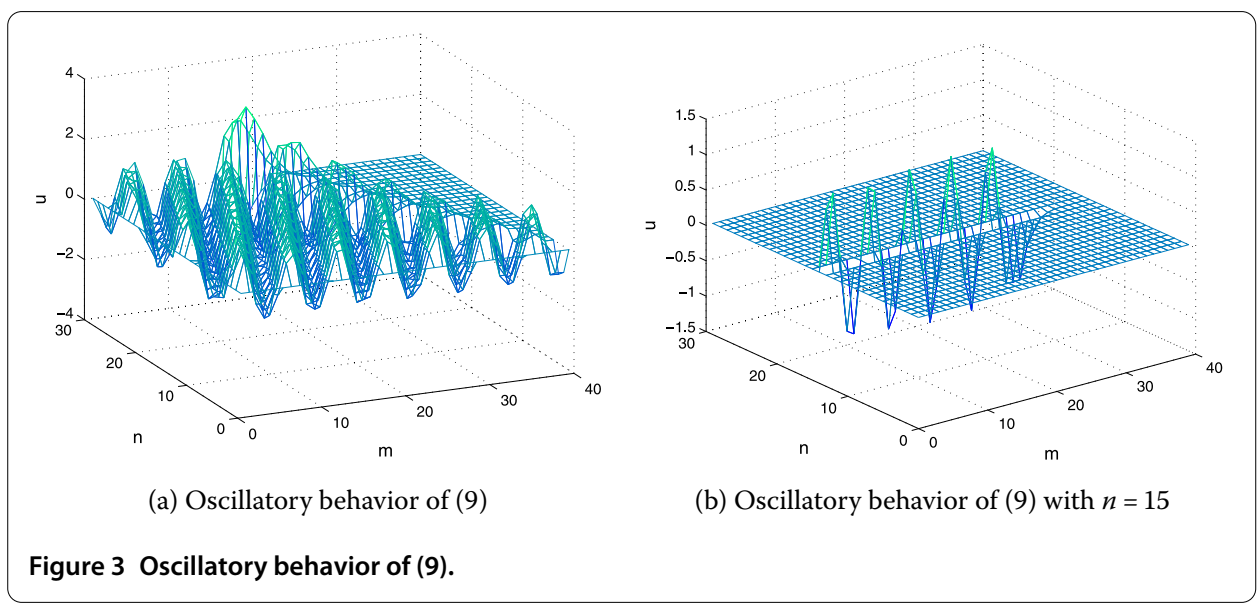

Example 2 Consider the partial difference equation

$$
u_{n+2, m}+u_{n, m+2}+0.02 u_{n+1, m}+0.12 u_{n, m+1}+0.12 u_{n, m}=0
$$

where $m, n$ are nonnegative integers.

From (9), we have $a=0.02>0, b=0.12>0$ and $c=0.12>0$. It follows from Theorem 1 that every solution of (9) is oscillatory. The oscillatory behavior of (9) is demonstrated by Figure 3.

\section{Conclusions}

In this paper, we have introduced a novel approach to problems of oscillations of a partial difference equation. Our approach is based on the envelope theory of the family of planes. We derive effective criteria to determine oscillations of a partial difference equation by this approach. Numerical examples are given to illustrate the results presented in this paper.

Competing interests

The authors declare that they have no competing interests. 


\section{Author details}

'School of Control Science and Engineering, Shandong University, Jinan, Shandong 250061, P.R. China. ${ }^{2}$ School of Mathematical Science, University of Jinan, Jinan, Shandong 250022, P.R. China.

\section{Acknowledgements}

This work is supported by the National Natural Science Foundation of P.R. China under Grant No. 61273088. The authors thank the editor and the referees for their valuable suggestions and comments.

\section{Received: 29 October 2014 Accepted: 26 January 2015 Published online: 28 March 2015}

\section{References}

1. Shi, BE, Chua, LO: Resistive grid image filtering: input/output analysis via the CNN framework. IEEE Trans. Circuits Syst. I 39, 531-548 (1992)

2. Meakin, P: Models for material failure and deformation. Science 252, 226-234 (1991)

3. Strikwerda, JC: Finite Difference Schemes and Partial Difference Equations. Wadsworth \& Brooks, Pacific Grove (1989)

4. Li, X: Partial difference equations used in the study of molecular orbits. Acta Chim. Sin. 40, 688-698 (1982)

5. Berman, G, Fryer, KD: Introduction to Combinatorics. Academic Press, New York (1972)

6. LeVeque, RJ: Numerical Methods for Conservation Laws. Birkhäuser, Berlin (1990)

7. Cheng, S: Partial Difference Equations. Advances in Discrete Mathematics and Applications, vol. 3. Taylor \& Francis, London (2003)

8. Yuan, C, Liu, S: An envelope surface method for determining oscillation of a delay 2-D discrete convection system. J. Differ. Equ. (2014). doi:10.1007/s10884-014-9422-x

9. Zhang, B, Xing, Q: Oscillation of certain partial difference equations. J. Math. Anal. Appl. 329, 567-580 (2007)

10. Zhang, B, Agarwal, RP: The oscillation and stability of delay partial difference equations. Comput. Math. Appl. 45 , 1253-1295 (2003)

11. Agarwal, RP, Zhou, Y: Oscillation of partial difference equations with continuous variables. Math. Comput. Model. 31, $17-29(2000)$

12. Zhang, B, Yu, J: Linearized oscillation theorems for certain nonlinear delay partial difference equations. Comput. Math. Appl. 35, 111-116 (1998)

13. Wong, PJY, Agarwal, RP: Oscillation criteria for nonlinear partial difference equations with delays. Comput. Math. Appl. 32, 57-86 (1996)

14. Zhang, B, Zhou, Y: Qualitative Analysis of Delay Partial Difference Equations. Hindawi Publishing Corporation, New York (2007)

15. Cheng, S, Lin, Y: Complete characterizations of a class of oscillatory difference equations. J. Differ. Equ. Appl. 2 , 301-313 (1996)

16. Cheng, S, Lin, Y: Complete characterizations of an oscillatory neutral difference equation. J. Math. Anal. Appl. 221, 73-91 (1998)

17. Chen, L, Lin, Y, Cheng, S: Exact regions of oscillation for a difference equation with six parameters. J. Math. Anal. Appl. 222, 92-109 (1998)

18. Cheng, S, Lin, Y, Rassias, TM: Exact regions of oscillation for a difference equation with five parameters. J. Differ. Equ. Appl. 6, 513-534 (2000)

19. Agarwal, RP, Grace, SR, O'Regan, D: Oscillation Theory for Difference and Functional Differential Equations. Kluwer Academic, Dordrecht (2000)

20. Cheng, S, Lin, Y: Dual Sets of Envelopes and Characteristic Regions of Quasi-Polynomials. World Scientific, Singapore (2009)

\section{Submit your manuscript to a SpringerOpen ${ }^{\ominus}$ journal and benefit from:}

- Convenient online submission

Rigorous peer review

Immediate publication on acceptance

- Open access: articles freely available online

- High visibility within the field

- Retaining the copyright to your article 